\title{
Ascomicetos de los guaduales en la reserva natural La Montaña del Ocaso, departamento del Quindío, Colombia
}

\author{
Ricardo Soto-Agudelo', Ricardo Valenzuela², Germán. D. Gómez-Marín', Tania Raymundo** \\ ${ }^{1}$ Centro de Estudios e Investigaciones en Biodiversidad y Biotecnología, Facultad de \\ Ciencias Básicas y Tecnologías, Universidad del Quindío, Armenia, Quindío, Colombia. \\ ${ }^{2}$ Instituto Politécnico Nacional, Escuela Nacional de Ciencias Biológicas, Departamento de Botánica, Laboratorio de Micología. \\ Plan de Ayala y Carpio s/n, Col. Santo Tomás, Delegación Miguel Hidalgo, Ciudad de México, México.
}

\begin{abstract}
Resumen
Se estudiaron 29 especies de ascomicetos recolectados en los guaduales de la Reserva Natural "La Montaña del Ocaso” del municipio de Quimbaya del departamento del Quindío en Colombia. De las especies encontradas, 15 crecen exclusivamente sobre restos en descomposición de Guadua angustifolia, 10 sobre madera en descomposición de árboles que crecen entre los guaduales y cuatro son parásitas de otros ascomicetos. De las especies encontradas, sólo Cookeina sulcipes ha sido mencionada para el Departamento del Quindío, las restantes 28 son citadas por primera vez para dicho departamento y de éstas, 19 son nuevos registros para Colombia. (C) 2016. Acad. Colomb. Cienc. Ex. Fis. Nat.
\end{abstract}

Palabras clave: Guadua angustifolia; Hypocreales; Orbiliales; Pezizales; Pleosporales; Xylariales.

Ascomycetes of the guadua forests in the natural reserve "La Montaña del Ocaso", Quindio department, Colombia

\begin{abstract}
Twenty nine species of Ascomycetes collected in the guadual from Natural Reserve of "Montaña del Ocaso" at the municipality of Quimbaya in the Quindío Department from Colombia are studied. Of these, 15 species only grow on decayed debris of Guadua angustifolia, 10 were found on decay wood of trees and four are parasites of others ascomycetes fungi. Of the collected species, only Cookeina sulcipes has been mentioned for Quindío Department, the remanining species are cited for the first time to this Department, and of these, 19 are new records from Colombia. (C) 2016. Acad. Colomb. Cienc. Ex. Fis. Nat.
\end{abstract}

Key words: Guadua angustifolia; Hypocreales; Orbiliales; Pezizales; Pleosporales; Xylariales.

\section{Introducción}

La Reserva Natural "La Montaña del Ocaso" se ubica en las coordenadas $4^{\circ} 34^{\prime} 08^{\prime \prime}$ ' LN y $75^{\circ} 51^{\prime} 03^{\prime \prime}$ LO dentro de la finca "El Ocaso" ubicada en la Vereda "El Laurel", al sur del municipio de Quimbaya (departamento del Quindío) a una altitud entre 975 y $1100 \mathrm{~m}$. El manejo técnico-científico de la reserva está a cargo de la Universidad del Quindío (Agudelo y Gómez, 2001). Los principales relictos de vegetación presentes en la Reserva Natural "La Montaña del Ocaso" son dominados por guaduales y un bosque andino. Los guaduales constituyen recursos florísticos de gran importancia ecológica por presentar condiciones de humedad, luz y temperatura que propician el desarrollo de un gran número de especies vegetales, animales y fúngicas. En este tipo de vegetación, la especie forestal dominante es Guadua angustifolia Kunth también conocida como "bambú colombiano" que es considerado el tercer bambú más grande del mundo y puede alcanzar hasta $30 \mathrm{~m}$ de altura (Agudelo y Gómez, 2001; Moreno-M, Trujillo y Osorio, 2007). Ramírez-Díaz y Camargo (2015) realizaron un estudio sobre la diversidad vegetal asociada a los guaduales y encontraron 67 familias y 197 géneros de plantas herbáceas, arbustivas y arbóreas, con las familias Moraceae, Rubiaceae, Solanaceae, Araceae y Lauraceae como las más predominantes. Guadua angustifolia es la especie nativa más importante de Colombia. Es un recurso natural que hasta hace pocos años sólo era utilizada para la construcción, pero hoy en día, se perfila como una nueva y excelente alternativa en la industria textil, construcción, artesanía, productos farmacéuticos, cosméticos naturales, alimentos, forraje industrial, y producción de pulpa y papel (Restrepo-F., Vélez-A., Botero-A. y Pulido-V., 2005). Por

\footnotetext{
*Correspondencia:

Tania Raymundo, traymundoo@ipn.mx

Recibido: 13 de marzo de 2016

Aceptado: 11 de julio de 2016
} 
otro lado, tenemos a los hongos del Phylum Ascomycota que son el grupo más diverso en el planeta con 64.163 especies (Kirk, Canon, Minter y Stalpers, 2008). Sin embargo, en Colombia se han registrado 181 especies de ascomicetos macroscópicos de acuerdo con el trabajo de Vasco-Palacios y Franco-Molano (2013) y de los microscópicos no se ha precisado la cifra. En el departamento del Quindío se han citado dos especies de ascomicetos Cookeina sulcipes y C. tricholoma por Franco-Molano en 2002 (fide VascoPalacios y Franco-Molano, 2013). Los estudios que registran especies de hongos asociadas a los guaduales son escasos, entre ellos tenemos el de Restrepo-F. et al. (2005), quienes aislaron y preservaron cuatro géneros de Agaricomycetes asociados al cultivo de guadua. El conocimiento sobre el bambú colombiano es basto, pero se conoce poco sobre los hongos asociados a ella, por lo que en el presente trabajo se tiene como objetivo determinar la diversidad taxonómica de los hongos del Phylum Ascomycota asociados a los guaduales y en particular a Guadua angustifolia en el departamento del Quindío en Colombia.

\section{Material y métodos}

En el presente trabajo se realizaron dos exploraciones en octubre y diciembre de 2015, en donde se recolectaron 81 especímenes de ascomicetos asociados a los guaduales y se determinaron 29 especies. Todo el material fue recolectado en la Reserva Natural “La Montaña del Ocaso” del municipio de Quimbaya del departamento del Quindío a una altitud de $1000 \mathrm{~m}$. Se registraron los datos de campo de los ejemplares recolectados como tamaño, forma, color del ascoma, así como el sustrato donde crecían y se les tomaron fotografías in situ con una cámara Nikon P510 y una Nikon D7000 con un lente DX Micro 85 mm; después, los ejemplares se deshidrataron, desinfectaron, etiquetaron y depositaron en el Herbario de la Universidad de Quindío(HUQ) con duplicados en la colección de hongos “Dr. Gastón Guzmán Huerta” del Herbario de la Escuela Nacional de Ciencias Biológicas del Instituto Politécnico Nacional (ENCB). En el laboratorio se realizaron preparaciones temporales en alcohol al $70 \%$ y $\mathrm{KOH}$ al 5\%, para observar y describir el tamaño, forma, color de las ascas, ascosporas, células estériles, así como la disposición de hifas o células del excipulo medular y ectal del apotecio, o paredes de los peritecios o pseudotecios. Los ejemplares se determinaron con las obras de Breintenbach y Kränzlin (1984), San Martín y Rogers (1989, 1995), Ju y Rogers (1996), Rogers y Ju (1998), Ju, Rogers, San Martín y Granmo (1998), Iturriaga y Pfister (2006), Chaverri, Huhndorf, Rogers y Samuels (2011), Liu, et al. (2011), Stadler, et al. (2014), Herrera, Rossman, Samuels, Liparini y Chaverri (2015). De las especies estudiadas, se indica con (*) a los nuevos registros para el departamento del Quindío y se da su distribución en Colombia, mientras que con $(* *)$ las especies que se citan por primera vez para Colombia y se incluye su descripción y distribución en el mundo. Se anexan láminas y fotografías in situ de las especies estudiadas.

\section{Resultados}

Dothideomycetes, Patellariales, Patellariaceae

**Rhytidhysteron rufulum (Spreng.) Speg., Anal. Soc. Cient. Argent. 90 (1-6): 177, 1921. Figura 1A

Histerotecios de 1-4 mm de largo y 0,5-1 mm de ancho, naviculares, alargados, rectos o flexuosos cuando jóvenes, con los extremos agudos, algunos son triradiados, negros, carbonáceos, errumpentes, superficiales, gregarios, raramente solitarios, cuando maduros o con la humedad abren de forma irregular a discoidal, tomando la forma apotecial, de 2-5 mm de diámetro, mostrando el epitecio de color anaranjado rojizo a negro al madurar y cambiando a rojo a magenta con $\mathrm{KOH}$, con los márgenes involutos. Surco longitudinal conspicuo, con el margen transversalmente estriado. Peridio de 40-72 $\mu \mathrm{m}$ de grosor, adelgazándose hacia el extremo basal y la parte media, parte apical más gruesa, carbonoso, glabro, compuesto por células seudoparenquimáticas globosas a subglobosas, de 9,6-12 $\times 8$-8,8 $\mu \mathrm{m}$, de paredes gruesas, de 1,6-2,4 $\mu \mathrm{m}$ de grosor. Pseudoparáfisis capitadas, septadas, hialinas, ramificadas hacia el ápice, de 1,6-2,4 $\mu \mathrm{m}$ de diám., fuertemente ensanchadas hacia el extremo apical, hasta 3,2-5,6 $\mu \mathrm{m}$ de ancho, fuertemente anastomosadas por encima de las ascas, formando un epitecio anaranjado, de 25,6-32 $\mu \mathrm{m}$ de grosor, el cual se torna violeta al reaccionar con $\mathrm{KOH} \mathrm{5 \% .} \mathrm{Ascas}$ de 152-199,5 × 12,2-15,2 $\mu \mathrm{m}$, bitunicadas, de paredes gruesas, cilíndricas, hialinas, octospóricas, con la base sinuosa. Ascosporas de 27,2-30,4 × 11,2-12 $\mu \mathrm{m}$, elipsoides a oblongas, ligeramente curvadas, uniseriadas, de pared gruesa y lisa, pigmentadas de marrón a marrón rojizo, con tres septos transversales, cingulados.

Material examinado. 29 octubre 2015, R. Soto-Agudelo 3 (HUQ); 29 diciembre 2015, R. Soto-Agudelo 67 (HUQ); T. Raymundo 5931 (HUQ, ENCB).

Distribución ecológica y geográfica. Esta especie crece gregaria en ramitas en descomposición de arbustos que crecen en los guaduales. Samuels y Müller (1979) indicaron que es una especie de amplia distribución en zonas tropicales y subtropicales de América. Murillo, Albertazzi, Carranza, Lumbsch y Tamayo (2009) lo citan de Costa Rica, e incluyen ejemplares de Estados Unidos, Puerto Rico y Venezuela. En México ha sido registrado por Méndez-Mayboca, Checa, Esqueda y Chacón (2010), Chacón, Tapia y Esqueda (2014) y Raymundo, et al. (2014) de bosque tropical seco. En este trabajo se cita por primera vez para Colombia.

\section{Pleosporales, Insertae sedis}

**Astrosphaeriella stellata (Pat.) Sacc., Syll. fung. 24 (2): 938, 1928. Figura 1B

Pseudotecios gregarios errumpentes, de 500-800 $\mu \mathrm{m}$ de diámetro, piriformes, de color marrón a vináceo oscuro con el ápice negro a completamente negros cuando maduros, textura carbonosa y superficie lisa con restos de sustrato en la base que le confiere una apariencia de estrella. Ostíolos papilados, prominentes. Peridio de 36-100 $\mu \mathrm{m}$ de grosor, 

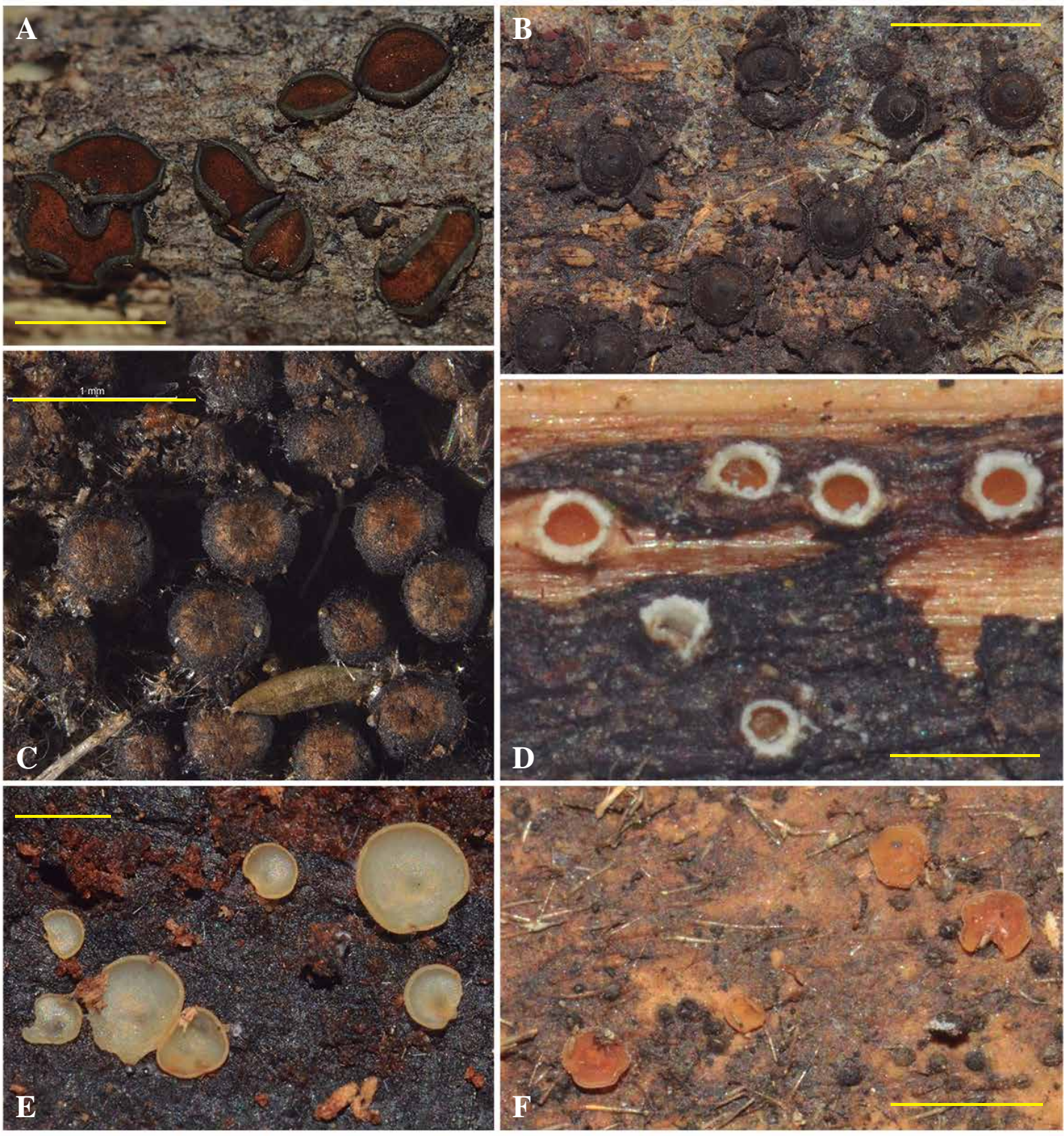

Figura 1. A-F: A. Rhytidhysteron rufulum, B. Astrosphaeriella stellata, C. Byssosphaeria rodomphala, D. Stictis radiata, E. Orbilia sarraziniana, F. O. xanthostigma (escala=1 mm).

poco desarrollado en la base. Pseudoparáfisis trabeculares, numerosas anastomosadas en las ramificaciones, hialinas, septadas, de hasta 1,2 de diám., embebidas en una matriz gelatinosa. Ascas de 180-225 × 12-14,6 $\mu \mathrm{m}$, bitunicadas, cilíndricas a clavadas, hialinas, octospóricas. Ascosporas de 44-52 × 5,6-7,2 $\mu \mathrm{m}$, fusiformes, con un septo central constreñido, hialinas cuando inmaduras a marrón pálido a marrón grisáceo al madurar y están rodeados por una vaina mucilaginosa, vaina truncada o redondeada en los extremos, desaparece con el $\mathrm{KOH}$, los extremos de las ascosporas adelgazados en el ápice, redondeados.

Material examinado. 29 diciembre 2015, R. SotoAgudelo 50, 72, 75, 78, 83 (HUQ); R. Valenzuela 16403, 16421, 16423 (HUQ, ENCB); T. Raymundo 5900, 5916, 5941 (HUQ, ENCB).
Distribución ecológica y geográfica. Esta especie crece sobre restos en descomposición de Guadua angustifolia. Citada de Taiwan por Chen y Hsieh (2004a) y de Australia, China, Filipinas, Francia, Guyana Francesa, India, Indonesia, Japón, Papúa Nueva Guinea, Tailandia y Vietnam por Liu et al. (2011). Se registra por primera vez para Colombia.

\section{Melanommataceae}

**Byssosphaeria rhodomphala (Berk.) Cooke, Grevillea 15 (no. 75): 81, 1887. Figura 1C

Pseudotecios superficiales, gregarios, de 350-650 $\mu \mathrm{m}$ de diámetro, globosos, subglobosos a turbinados, colapsándose con la edad, con ostíolo de color anaranjado brillante a anaranjado ladrillo, con estrías o surcos que rodean el poro circular, la base es oscura a negra. Subículo bien 
desarrollado, formado por un tomento negro. Peridio de 25-80 $\mu \mathrm{m}$ de grosor, formado por células isodiamétricas, pseudoparenquimatosas, rojizas en la parte apical, al contacto con $\mathrm{KOH}$ al 5\% desprende una coloración vinácea. Pseudoparáfisis trabeculares, hialinas, de 1-2 $\mu \mathrm{m}$, de diám. Ascas de $78-110 \times 10-12 \mu \mathrm{m}$, bitunicadas, clavadas a cilíndricas, octospóricas. Ascosporas de 20-22 × 6,4-7,2 $\mu \mathrm{m}$, bicelulares, con septo medio constreñido, fusoides, de color marrón amarillento, con los extremos obtusos.

Material examinado. 29 octubre 2015, R. Soto-Agudelo 44 (HUQ).

Distribución ecológica y geográfica. Esta especie se desarrolla sobre madera en descomposición de una leguminosa (Fabaceae). Citada de Cuba, EUA, Panama, Trinidad (Barr, 1984), Brasil (Réblová, 1997), Taiwan (Chen y Hsieh, 2004b) China (Li y Zhuang, 2008), México, Puerto Rico (Chacón-Zapata y Tapia-Padilla, 2013) y Argentina (Gallo, Robledo, Romero y Catania, 2014), todos mencionan que crece sobre madera en descomposición. No citada previamente de Colombia.

Lecanoromycetes, Ostropales, Stictidaceae

*Stictis radiata (L.) Pers., Observ. mycol. 2: 73, 1800. Figura 1D

Material examinado. 29 octubre 2015, R. Soto-Agudelo 46 (HUQ); 29 diciembre 2015, R. Soto-Agudelo 52 (HUQ); R. Valenzuela 16404, 16413, 16414 (HUQ, ENCB); T. Raymundo 5902 (HUQ, ENCB).

Distribución ecológica y geográfica. Crece en restos en descomposición de Guadua angustifolia. Se conoce previamente de Colombia del Parque Nacional Los Nevados (Boekhout y Pulido, 1989; Pulido y Boekhout, 1989). En este trabajo se cita por primera vez para el Quindío.

\section{Orbiliomycetes, Orbiliales, Orbiliaceae}

**Orbilia sarraziniana Boud., Revue mycol. 7: 221, 1885. Figura 1E

Apotecios discoide de 300-850 $\mu \mathrm{m}$ de diámetro, turbinado a discoide, sésiles, de color pálido amarillento a rosado en fresco a anaranjado en seco, traslucido. Excípulo ectal de $50 \mu \mathrm{m}$ de grosor, textura angular, hialino en $\mathrm{KOH}$, con células angulares de 11-14 $\mu \mathrm{m}$ de diámetro, pared delgada, pigmentos amarillentos. Células marginales corticales con textura globulosa, células de hasta $20 \mu \mathrm{m}$ de diámetro. Excípulo medular de $60 \mu \mathrm{m}$ de grosor, de color pálido amarillo a ocráceo, textura intricata. Paráfisis hialinas, cilíndricas, ápice fuertemente clavado-capitado, de 2-4 $\mu \mathrm{m}$ de diám. en el ápice y no rebasan la longitud del asca. Ascas de (30-) 38-40 (-46) × 2-3 $\mu \mathrm{m}$, hialinas, octospóricas, biseriadas. Ascosporas de (7-) 8-10 × $1 \mu \mathrm{m}$, cilíndricas a fusiformes, ápice agudo, base ligeramente atenuadas, hialinas, de paredes delgadas y lisas.

Material examinado. 29 diciembre 2015, T. Raymundo 5910 (HUQ, ENCB).

Distribución ecológica y geográfica. El espécimen fue recolectado sobre estromas de Annulohypoxylon multiforme, y crece como parásito de esta especie. Citada de Suiza por Breintenbach y Kränzlin (1984). No se conocía de Colombia.

**Orbilia xanthostigma (Fr.) Fr., Summa veg. Scand., Section Post.: 357, 1849. Figura 1F

Apotecios discoide de 300-350 $\mu \mathrm{m}$ de diám., cupuliformes a discoidales, sésiles, de color anaranjado rojizo, traslucido en fresco, marrón anaranjado en seco. Excípulo ectal de $50 \mu \mathrm{m}$ de grosor, textura angular, de color amarillo pálido en $\mathrm{KOH}$, con células angulares de 11-14 $\mu \mathrm{m}$ de diámetro, pared delgada, pigmentos amarillentos. Células marginales corticales de textura globulosa, hasta de $16 \mu \mathrm{m}$ de diámetro. Excípulo medular de $60 \mu \mathrm{m}$ de grosor, de color pálido amarillo a ocráceo, textura intricata. Paráfisis hialinas, cilíndricas, ápice ligera a fuertemente clavado-capitado, de 1-3 $\mu \mathrm{m}$ de diámetro en el ápice. Ascas de (30-) 38-40 (-46) $\times 2-3 \mu \mathrm{m}$, hialinas, octospóricas, biseriadas. Ascosoporas de (6-) 8-10 $\times 1 \mu \mathrm{m}$, cilíndricas a alantoides, ápice agudo, ligeramente atenuadas en la base, hialinas, de paredes delgadas y lisas.

Material examinado. 29 diciembre 2015, T. Raymundo 5930 (HUQ, ENCB).

Distribución ecológica y geográfica. Creciendo sobre estromas de un hongo de la familia Diatrypaceae sobre una rama muerta de angiosperma. Esta especie ha sido citada de Suiza por Breintenbach y Kränzlin (1984). En el presente trabajo se menciona por primera vez para Colombia.

Pezizomyctes, Pezizales, Pezizaceae

*Cookeina speciosa(Fr.) Dennis, Mycotaxon 51: 239, 1994. Figura 2G

Material examinado. 29 diciembre 2015, R. SotoAgudelo 51 (HUQ); R. Valenzuela 16402 (HUQ, ENCB); $T$. Raymundo 5901 (HUQ, ENCB).

Distribución ecológica y geográfica. La especie fue recolectada en restos de tallos en descomposición de Guadua angustifolia. En Colombia fue citado por Guzmán, Torres, Ramírez-Guillen y Ríos-Hurtado (2004), Franco-Molano et al. (2005), Vasco-Palacios, et al. (2005) y Betancour, M, Calderón, Betancourt, O y Sucerquia (2007) de los departamentos de Amazonas, Antioquia, Choco, Caldas y Caquetá creciendo sobre madera en descomposición de angiospermas. No citada previamente en el Quindío.

Cookeina sulcipes (Berk.) Kuntze, Revis. gen. pl. 2: 849, 1891. Figura 2H

Material examinado. 29 octubre 2015, T. Raymundo 5899 (HUQ, ENCB).

Distribución ecológica y geográfica. La especie se localizó en restos de Guadua angustifolia. En Colombia ha sido citado por Franco-Molano en 2002 (fide Vasco-Palacios y Franco-Molano, 2013) del Quindío, y por FrancoMolano, Vasco-Palacios, López-Quintero y Boekhout (2005), Vasco-Palacios, et al. (2005) de los departamentos de Amazonas, Antioquia, Choco, Caldas y Caquetá sobre madera en descomposición de otros hospederos. 

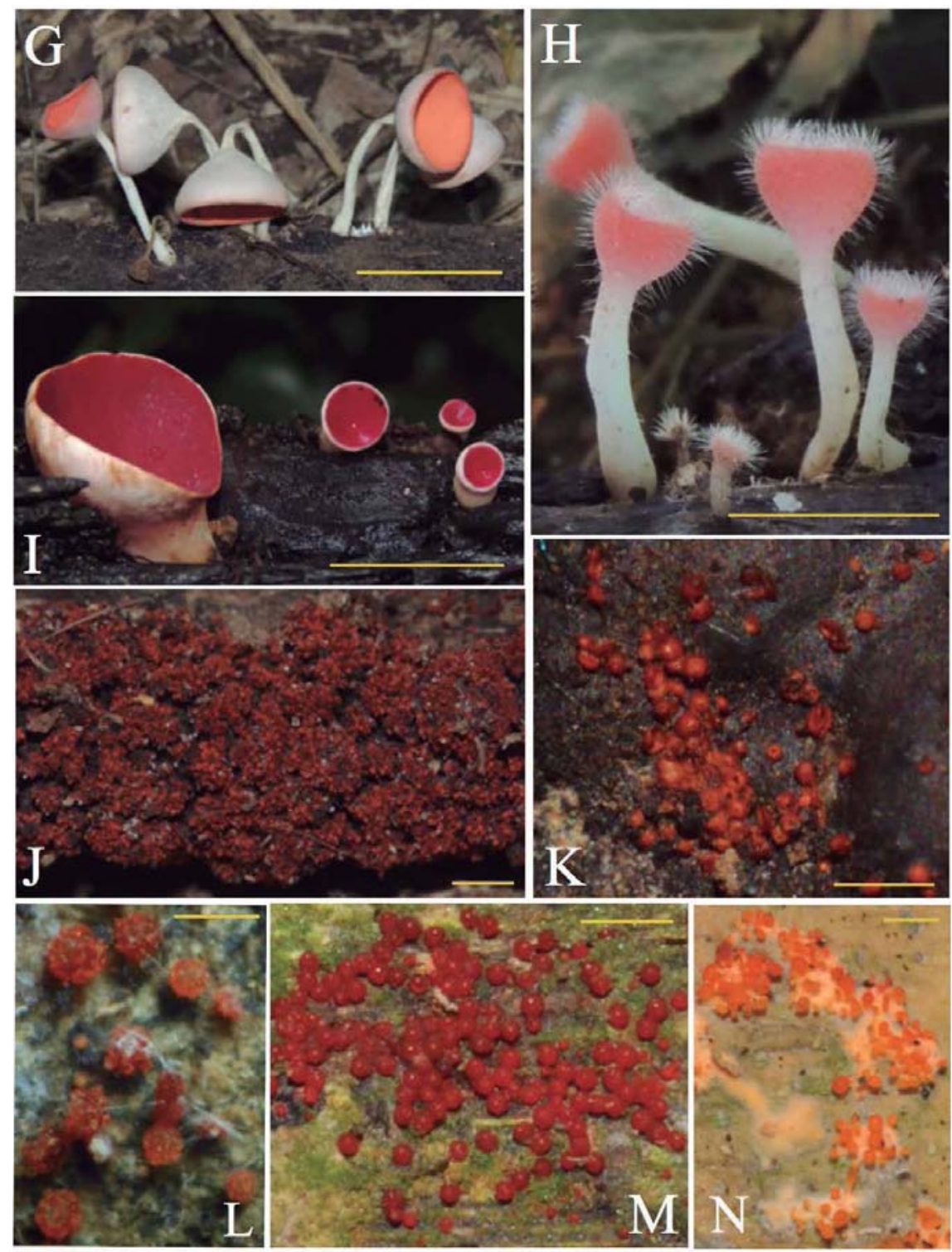

Figura 2. G-N: G. Cookeina speciosa, H. C. sulcipes, I. Phillipsia domingensis, J. Cosmospora annulohypoxili, K. C. ustulinae, L. Haematonectria haematococca, M. Thelonectria jungneri, N. Hypocreopsis macrostoma. (escala G-I=5 cm, J-N=1mm).

*Phillipsia domingensis Berk., J. Linn. Soc. Bot. 18: 388, 1881. Figura 2I

Material examinado. 29 octubre 2015, R. Soto-Agudelo 9 (HUQ).

Distribución ecológica y geográfica. Fue recolectada en madera en descomposición de árboles que crecen entre los guaduales. En Colombia ha sido mencionada en el departamento de Caquetá (Franco-Molano, et al., 2005; Vasco-Palacios, et al., 2005). En este trabajo se registra por primera vez para el Quindío.

\section{Sordariomycetes, Hypocreomycetidae, Hypocreales, Nectriaceae}

**Cosmospora annulohypoxyli C. Herrera \& P. Chaverri, in Herrera, et al. Mycologia 107: 545, 2015. Figura 2J
Peritecios de 192-200 × 175-200 $\mu \mathrm{m}$, subglobosos, con ápices redondeados de 80-85 × 35-40 $\mu \mathrm{m}$, de superficie lisa y colapsados lateralmente cuando secos, de color rojo, cambian a amarillo en ácido láctico; gregarios a solitarios sobre el hospedero. Pared del peritecio de $20 \mu \mathrm{m}$ de diámetro. Ascas de 50-60 × 5-5.5 $\mu \mathrm{m}$, octospóricas, cilíndricas, uniseriadas, hialinas en $\mathrm{KOH} \mathrm{5 \%}$. Ascosporas de (5.6-) 6.4-7 × (2.4-) 3-4 $\mu \mathrm{m}$, elipsoidales, con septo en la parte media, hialinas, verrucosas en azul de algodón y lactofenol.

Material examinado. 29 diciembre 2015, R. Valenzuela 16400 (HUQ, ENCB).

Distribución ecológica y geográfica. Fue encontrada creciendo sobre estromas de Annulohypoxylon multiforme, de la cual es parásita. Herrera, et al. (2015) describieron recientemente esta especie para Louisiana, EE UU y 
mencionan que solo crece sobre Annulohypoxylon coharens y A. multiforme. Esta especie se describe por primera vez en Colombia.

**Cosmospora ustulinae (Teng) C. Herrera \& P. Chaverri, in Herrera, et al. Mycologia 107: 550, 2015. Figura 2K

Peritecios de 209-228 $\mu \mathrm{m}$ de alto y 171-199.5 $\mu \mathrm{m}$ de diámetro, piriforme, de color rojo, estroma inconspicuo. Cuello de 95-110.2 $\mu \mathrm{m}$ de alto y 28.5-43.7 $\mu \mathrm{m}$ de diámetro, en ocasiones se pueden observar las perífisis emergiendo del ostíolo inconspicuo a simple vista. Pared de 18-20 $\mu \mathrm{m}$ de diámetro, tornándose rojo oscuro con $\mathrm{KOH}$ al 5\% y naranja o amarillento con ácido láctico, lisa; células de 8-12 $\mu \mathrm{m}$ de largo y 2,4-4,8 $\mu \mathrm{m}$ de diámetro, formando textura epidermoidea. Ascas de 50,4-57,6 × 5,4-5,6 $\mu \mathrm{m}$, cilíndricas, hialinas, sin aparato apical aparente, conteniendo ocho ascosporas dispuestas de forma uniseriada. Ascosporas de 6,4-8,8 $\times$ 4-5 $\mu \mathrm{m}$, elipsoides, constreñidas por un septo central, hialinas a amarillentas, verrugosas en azul de algodón y lactofenol.

Material examinado. 29 octubre 2015, R. Soto-Agudelo 11 (HUQ); 29 diciembre 2015, R. Soto-Agudelo 53 (HUQ); R. Valenzuela 16405 (HUQ, ENCB); T. Raymundo 5903 (ENCB).

Distribución ecológica y geográfica. Los especímenes colombianos fueron encontrados sobre estromas de Kretzschmaria pavimentosa (Hoffm.) P.M.D. Martin. Esta especie se ha citado de China, Japón, Portugal y Estados Unidos de América por Herrera, et al. (2015). Se registra por primera vez para Colombia.

*Haematonectria haematococca (Berk. \& Broome) Samuels \& Rossman, in Rossman, Samuels, Rogerson \& Lowen, Stud. Mycol. 42: 135, 1999. Figura 2L

Peritecios de 300-377 $\mu \mathrm{m}$ de diámetro, globosos a piriformes, de color rojo a naranja rojizo, colapsados lateralmente cuando secos, cambiando a rojo oscuro en $\mathrm{KOH} 5 \%$ y amarillo en ácido láctico, la superficie está cubierta de escamas de color naranja; solitarios a gregarios, superficiales en el sustrato. Ostíolo inconspicuo, perífisis de 58-60 × 0,8-1 $\mu \mathrm{m}$, curvadas en el ápice, hialinas en $\mathrm{KOH} 5 \%$. Pared del peritecio de 70-90 $\mu \mathrm{m}$ de grosor, con dos capas de células, la externa formada por células de 1926,4 $\mu \mathrm{m}$ de diámetro, formando textura globulosa y las escamas en vista macroscópica, de pared gruesa, hialinas y amarillentas en $\mathrm{KOH} \mathrm{5 \% .} \mathrm{La} \mathrm{capa} \mathrm{interna} \mathrm{está} \mathrm{formada}$ por células de 15-25,6 $\mu \mathrm{m}$ de diámetro, de pared delgada, formando textura epidermoidea a casi globulosa. Ascas de 80-84 × 6.9-7.2 $\mu \mathrm{m}$, con estípite largo,octospóricas, cilíndricas a clavadas, con un anillo apical visible en ácido láctico, hialinas. Ascosporas de 12,8-14,4 × 5,6-6,4 $\mu \mathrm{m}$, apicalmente biseriadas, elipsoides, con un septo, hialinas y finamente estriadas.

Material examinado. 29 octubre 2015, R. Soto-Agudelo 3 (HUQ).

Distribución ecológica y geográfica. La especie crece en ramas recién caídas de Guadua angustifolia. Se distribuye en la región pantropical (Chaverri, et al., 2011). Fue registrado de los departamentos de Antioquia, Cundinamarca y Valle del Cauca en Colombia por Samuels y Dumont (1982). Se cita por primera vez para el Quindío.

**Thelonectria jungneri (Henn.) P. Chaverri \& C. Salgado, in Chaverri, Salgado, Hirooka, Rossman \& Samuels, Stud. Mycol. 68: 76, 2011. Figura 2M

Peritecios de 539-623,7 × 308-469,7 $\mu \mathrm{m}$, obpiriformes, similares a jarrones, superficiales, de color rojo cuando fresco, no colapsados en su mayoría cuando secos aunque en ocasiones colapsados lateralmente, color rojo oscuro en $\mathrm{KOH} 5 \%$ y amarillo en ácido láctico, superficie lisa a ligeramente rugosa, gregarios. Ostíolo levemente papilado, de 135-180 $\mu \mathrm{m}$ de diámetro, del mismo color que el resto del peritecio o bien negruzco a casi morado. Pared de 25$38 \mu \mathrm{m}$ de diámetro Ascas de 70-97 × 12-17 $\mu \mathrm{m}$, clavadas, octospóricas, biseriadas, hialinas, anillo apical visible en ácido láctico. Ascosporas de 26,4-28,8 × 11,2 $\mu \mathrm{m}$, fusiformes, con un septo en la parte media, hialinas, con marcadas estriaciones longitudinales visibles en $\mathrm{KOH}$.

Material examinado. 29 octubre 2015, R. Soto-Agudelo 8 (HUQ).

Distribución ecológica y geográfica. Fue recolectada sobre restos en descomposición de Guadua angustifolia. Esta especie se ha citado de Japón (Hirooka y Kobayashi 2007), de Taiwan (Guu, Ju y Hsieh, 2007), de Costa Rica (Chaverri, et al., 2011) y de México (Raymundo, et al., 2014). En este trabajo se registra por primera vez para Colombia.

\section{Hypocreaceae}

**Hypocreopsis macrostoma (Berk. \& M.A. Curtis) E. Müll., in Müller \& von Arx, Beitr. Kryptfl. Schweiz 11 (no. 2): 650, 1962. Figura $2 \mathbf{N}$

Estromas de 1-10 × 1-12 mm de ancho y de 400-1200 $\mu \mathrm{m}$ de alto, bien desarrollados, pulvinados, convexos o irregulares, de color naranja rosado, formados por hifas de 4-5 $\mu \mathrm{m}$ de diámetro, formando textura intricata, hialinas a amarillentas en $\mathrm{KOH}$ 5\%. Peritecios de 140,6-207,9 × 98,8$177,1 \mu \mathrm{m}$, globosos a subglobosos, completamente inmersos en el estroma, a excepción del ostiolo. Ostíolos apenas visibles, de 70-80 $\mu \mathrm{m}$ de diámetro, color rosa brillante. Pared del peritecio de 9,6-21,6 $\mu \mathrm{m}$ de diámetro, con células de 7,2-8 × 2,4-3 $\mu \mathrm{m}$, formando textura prismática a casi epidermoidea, con paredes delgadas, de color amarillo dorado. Ascas de 70-74,4 × 6-7,2 $\mu \mathrm{m}$, octospóricas, cilíndricas, con anillo apical. Ascosporas de 10,4-11,2 × 4,8 $\mu \mathrm{m}$, uniseriadas, elipsoides a ligeramente fusiformes, con un septo en el centro, hialinas, ligeramente rugosas o verrucosas en ácido láctico. Su estado asexual era conocido como Stilbella aleuriata (Berk. \& M.A. Curtis) Seifert, forma sinemas de 500-1750 × 80-135 $\mu \mathrm{m}$, solitarios, de color amarillo-naranja, rectos a ligeramente curvados, con masa conidial en la parte apical. Conidióforos colocados en la parte apical del sinema, ramificados, con 2 a 3 fiálides de $12-20 \times 1-2 \mu \mathrm{m}$; conidios unicelulares de 2,4-4 × 1,6-2,4 $\mu \mathrm{m}$, oblongos a elipsoidales, hialinos. 
Material examinado. 29 diciembre 2015, R. SotoAgudelo 57, 58, 79 (HUQ); R. Valenzuela 16408, 16409 (HUQ, ENCB); T. Raymundo 5913, 5914, 5915 (HUQ, ENCB).

Distribución ecológica y geográfica. Esta especie fue recolectada tanto en tallos vivos como recién caídos de Guadua angustifolia. Citada de Taiwán (Guu, et al., 2010), de Costa Rica (Chaverri, et al., 2011) y de México (Raymundo, et al., 2014). No se conocía en Colombia.

*Trichoderma patella (Cooke \& Peck) Jacklitsch \& Voglmayr, Mycotaxon 126: 151, 2014. Figura 3Ñ

Estroma pulvinado de 1100-1350 × 680-770 $\mu \mathrm{m}$, superficie plana, peritecios poco evidentes o sobresaliendo el ostíolo a manera de papilas. Capa superficial del estroma de 48-56 $\mu \mathrm{m}$ de grosor, células marrón rojizas, subglobosas a angulares, de 8-9,6 $\mu \mathrm{m}$, de diámetro, paredes delgadas a gruesas, de hasta $1.5 \mu \mathrm{m}$ de grosor. Peritecios de 162-170 × 126$153 \mu \mathrm{m}$; peridio de $24-40 \mu \mathrm{m}$ de grosor, con células marrón rojizas, subglobosas, de 4-4,5 $\mu \mathrm{m}$ de diámetro. Ascas de 67.2$72 \times 4-4.4 \mu \mathrm{m}$, cilíndricas, hialinas, octospóricas. Ascosporas uniseriadas, bicelulares, hialinas, finamente espinulosas, con la parte distal globosa, de 2,8-4 × 2,8-3.2 $\mu \mathrm{m}$, y la parte proximal subglobosa a oblonga, de 3,6-4,8 $\times 2,8-3.2 \mu \mathrm{m}$.

Material examinado. 29 octubre 2015, R. Valenzuela 16397 (HUQ).

Distribución ecológica y geográfica. La especie se encontró en tallos vivos de Guadua angustifolia. Chaverri, et al. (2011) la citaron de Costa Rica y Colombia. Se cita por primera vez del departamento del Quindío.

Sordariomycetidae, Chaetosphaeriales, Chaetosphariaceae ** Chaetosphaeria lapaziana (Carroll \& Munk) F.A. Fernández \& Huhndorf, Fungal Diversity 18: 49, 2005. Figura 30

Peritecios de 600-1000 × 400-800 $\mu \mathrm{m}$, ovoides a piriformes, marrón oscuro a negros y lisos. Pared gruesa de $50-90 \mu \mathrm{m}$ de grosor, con textura globosa formada de 1218 capas de células pseudoparenquimáticas a angulares, isodiamétricas de 30-40 $\mu \mathrm{m}$ de diámetro, marrón pálido y liberando pigmentos purpuras en $\mathrm{KOH}$ al 5\%. Ostíolo de 35$80 \mu \mathrm{m}$ de diám. Paráfisis de 3-4 $\mu \mathrm{m}$ de diámetro, septadas y hialinas. Ascas de 160-280 × 12-24 $\mu \mathrm{m}$, cilíndricas con el ápice redondeado, octospóricas y triseriadas. Ascosporas de 45-90 × 4,6-6,8 $\mu \mathrm{m}$, filiformes a cilíndricas, extremos redondeados, heptaseptadas, ligeramente curvadas, hialinas, de pared delgada, lisas.

Material examinado. 29 diciembre 2015, T. Raymundo 5943 (HUQ, ENCB).

Distribución ecológica y geográfica. Esta especie se recolectó sobre tallos en descomposición de Guadua angustifolia. Se ha citado de Costa Rica, Guyana Francesa, Jamaica y Puerto Rico (Fernández y Huhndorf, 2005; Chaverri, et al., 2011). En este trabajo se menciona por primera vez para Colombia.

\section{Xylariales, Diatrypaceae}

**Diatrype bermudensis Rappaz, Mycologia Helvetica 2:440, 1987. Figura 3P-Q
Estromas de 4-12 mm de largo y 0,5-1 (-2) mm de ancho, lineares, forma hileras múltiples paralelas al tallo, superficie rugosa o granulosa por los cuellos y ostíolos de los peritecios errumpentes, solitarios o confluentes, de color gris oscuro a negro. Peritecios de 400-550 $\times 200-300 \mu \mathrm{m}$, globosos a subglobosos, con el cuello de 150-180 × 50-70 $\mu \mathrm{m}$, cilíndrico, proyectándose ligeramente de la superficie del estroma. Ascas de 28-42 × 6-8 $\mu \mathrm{m}$, cilíndricas a claviformes, pedicelada, con un base delgada alargada, octospóricas. Ascosporas de 9-12 × 2-2.5 $\mu \mathrm{m}$, irregularmente dispuestas, unicelulares, alantoides a fuertemente curvadas, amarillentas a marrón pálido en $\mathrm{KOH}$, de paredes delgadas, lisas.

Material examinado. 29 diciembre 2015, R. SotoAgudelo 66 (HUQ); T. Raymundo 5925, 5940 (ENCB); $R$. Valenzuela 16415, 16422 (ENCB).

Distribución ecológica y geográfica. La especie fue recolectada en la base de tallos vivos o muertos de Guadua angustifolia. Rappaz (1987) la describió de Bermudas sobre bambú muertos y Chacón, Dörge, Weisenborn y Piepenbring (2013) de Panamá creciendo en tallos muertos de Guadua angustifolia. No registrada previamente de Colombia.

\section{Xylariaceae}

*Annulohypoxylon multiforme (Fr.) Y.M. Ju, J.D. Rogers \& H.M. Hsieh, Mycologia 97(4): 859, 2005. Figura 3R

Material examinado. 29 diciembre 2015, R. SotoAgudelo 69 (HUQ); R. Valenzuela 16419 (ENCB); T. Raymundo 5929 (ENCB).

Distribución ecológica y geográfica. Se encontró tanto en tallos vivos como recién caídos de Guadua angustifolia. Esta especie fue citado del Valle del Cauca por Chardon \& Toro en 1930 (fide Vasco-Palacios y Franco-Molano, 2013). Se menciona por primera vez para el Quindío.

**Camillea coroniformis J. D. Rogers, F. San Martín \& Y. M. Ju, Sydowia 54: 87, 2002. Figura 3 S

Estroma de 20-50 × 20-30 mm, circular, elipsoidal a irregular en forma, errumpente, aplanado en la superficie, de color negro brillante, consistencia carbonosa. Peritecios de $700 \times 300 \mu \mathrm{m}$, tubulares, completamente inmersos en el estroma. Ascas de 120-135 × 7-9 $\mu \mathrm{m}$, con anillo apical amiloide, octospóricas. Ascosporas de 14-16 × 5-6 $\mu \mathrm{m}$, elipsoidales, hialinas, con ápices agudos, sin línea germinal.

Material examinado. 29 diciembre 2015, R. SotoAgudelo 55; T. Raymundo 5908 (HUQ).

Distribución ecológica y geográfica. Fue recolectada en árboles recién caídos de angiospermas en los guaduales. Citada de Costa Rica (Rogers, San Martín y Ju, 2002) y de México (Raymundo, et al., 2014). Se registra por primera vez para Colombia.

*Daldinia eschscholtzii (Ehrenb.) Rehm, Annls Mycol. 2(2): 175, 1904. Figura 3T

Material examinado. 29 octubre 2015, T. Raymundo 5897 (HUQ). 

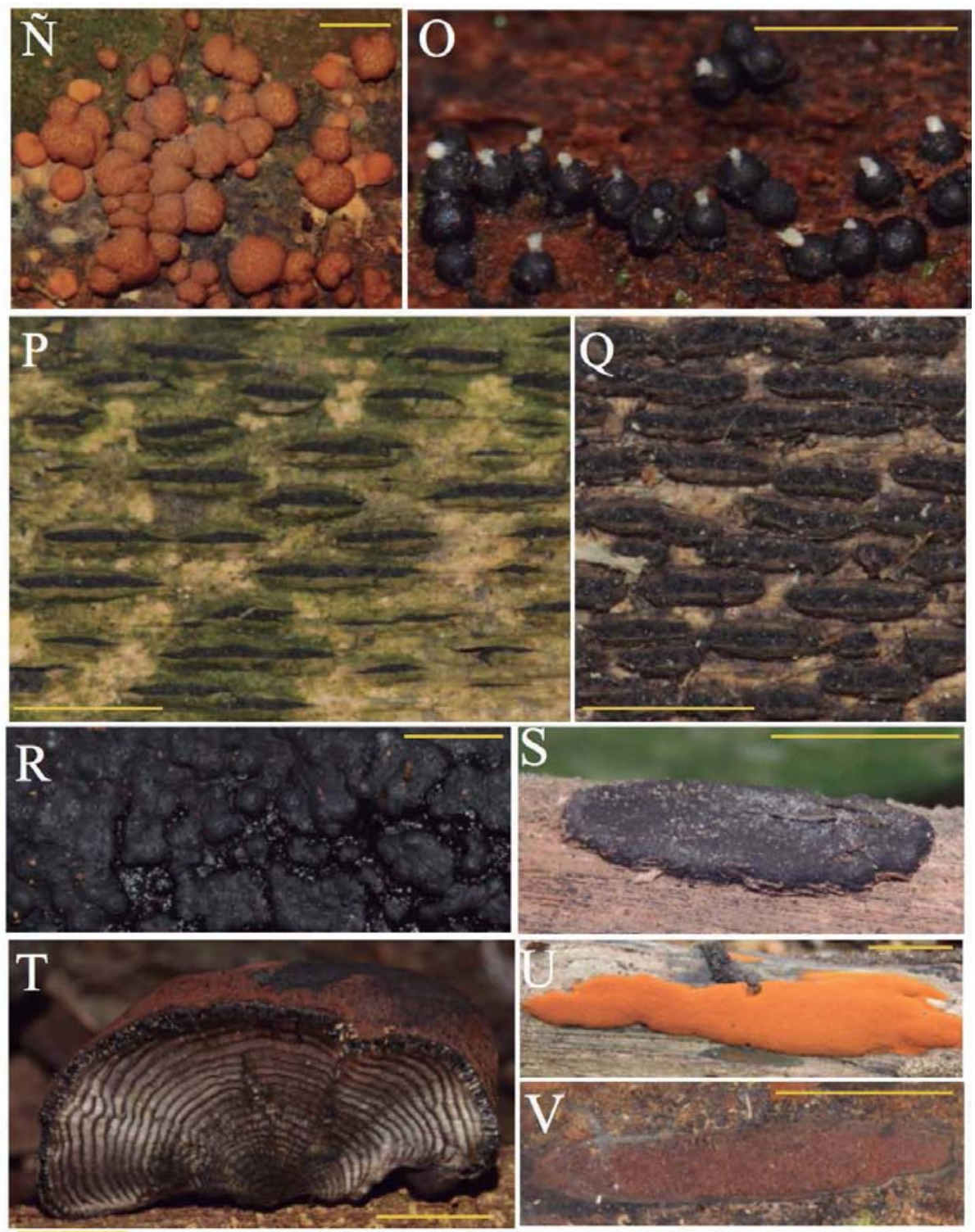

Figura 3. Ñ-V: Ñ. Trichoderma patella, O. Chaetosphaeria lapaziana, P-Q. Diatrype bermudensis, R. Annulohypoxylon multiforme, S. Camillea coroniformis, T. Daldinia eschscholtzii, U. Hypoxylon haematostroma V. Hypoxylon investiens. $($ escala $=2 \mathrm{~cm})$.

Distribución ecológica y geográfica. Esta especie se encontró solitaria o gregaria en madera en descomposición de árboles en los guaduales. En Colombia ha sido registrada del Chocó por Guzmán, et al. (2004) y de Antioquia por Stadler, et al. (2014). No había sido mencionada para el Quindío.

**Hypoxylon haematostroma Mont., in Sagra, Annls Sci. Nat., Bot., sér. 2, 17: 124, 1842. Figura 3U

Estroma de $300-500 \times 200-400 \times 1.5-3 \mathrm{~mm}$, plano a efuso-pulvinado, con peritecios inconspicuos a poco conspicuos, superficie de color naranja brillante con gránulos rojos, a naranja oscuro al madurar, pigmentos extraíbles en $\mathrm{KOH}$ de color escarlata a naranja y marrón rojizo en ejemplares más viejos. El tejido entre los peritecios es de color negro, de 500-1000 $\mu \mathrm{m}$ de grosor. Peritecios de $1000-2500 \times 300-600 \mu \mathrm{m}$, tubulares, de color negro, con ostíolos por debajo de la superficie del estroma. Ascas de 80-120 × 9-11 $\mu \mathrm{m}$, con pedicelos de 160-220 $\mu \mathrm{m}$, con anillo apical bien desarrollado, de 1,4-2 × 3-4 $\mu \mathrm{m}$, discoide, amiloide, octospóricas. Ascosporas de 14-18 × 6-8,4 $\mu \mathrm{m}$, elipsoidales con los extremos ampliamente redondeados, marrón obscuro en $\mathrm{KOH}$, poro germinal recto con perisporio dehiscente y episporio liso.

Material examinado. 29 octubre 2015, T. Raymundo 5898 (HUQ).

Distribución ecológica y geográfica. Crece solitaria o gregaria en madera en descomposición de árboles en los guaduales. Es una especie de amplia distribución en las zonas tropicales (Ju y Rogers, 1996). Se cita por primera vez para Colombia. 
*Hypoxylon investiens (Schwein.) M. A. Curtis, Goel. Nat. Hist. Surv. N. Carol., Pt 3: 140, 1867. Figura 3V

Material examinado. 29 diciembre 2015, R. SotoAgudelo 56, 80, 81, 82 (HUQ); R. Valenzuela 16407 (ENCB); T. Raymundo 5909, 5919, 5932, 5934, 5937 (ENCB).

Distribución ecológica y geográfica. Se recolectó en tallos en descomposición de Guadua angustifolia. Esta especie fue citada de Colombia del Parque Nacional Natural Los Nevados por Boekhout y Pulido (1989), Pulido y Boekhout (1989). Se registra por primera vez en el Quindío.

**Hypoxylon leneormandii Berk. \& M.A. Curtis apud. Berk., J. Linn. Soc., Bot. 10 (46): 385, 1868. Figura 4W

Estroma de 200-350 × 80-150 mm, aglomerado a efusopulvinado, ocasionalmente se observan peritecios solitarios tipo rosselinoide pero unidos por un subículo o estroma muy delgado y fino. Peritecios conspicuos de 400-970 $\times$ 300-780 $\mu \mathrm{m}$ de diámetro, globosos a subglobosos, de color marrón oscuro a marrón grisáceo, con pigmentos extraíbles en $\mathrm{KOH}$, de color canela, marrón rojizo a ocráceo en ejemplares más viejos, con ostiolos agudos de color negro. Ascas de 124-160 × 7-9 $\mu \mathrm{m}$, cilíndricas, con pedicelos de 32-72 $\mu \mathrm{m}$ de largo, con anillo apical, de 0,8-1,4 × 2-3 $\mu \mathrm{m}$, discoide, amiloide, octospóricas. Ascosporas de 10,4-15 $\times$ 4-4,8 (-6) $\mu \mathrm{m}$, elipsoides, con los extremos redondeados, marrón oscuro, poro germinal ligeramente sigmoide, con perisporio dehiscente y episporio liso.

Material examinado. 29 dic 2015, R. Valenzuela 16401; T. Raymundo 5907 (HUQ).

Distribución ecológica y geográfica. Crece en tallos en descomposición de Guadua angustifolia. Registrada de Norteamerica (Ju y Rogers, 1996), México (San Martín, Ju y Rogers, 1999; Raymundo, et al., 2014) y Panamá (Carmona, Fournier, Williams y Piepenbring, 2009). No citada previamente de Colombia.

**Kretzschmaria pavimentosa (Ces.) Cooke, Jl S. Afr. Bot. 42(1): 74, 1976. Figura 4X

Estromas de $100-380 \times 25-40 \mathrm{~mm}$, completamente adherido al sustrato, pulvinados, de color marrón cobrizo a marrón oscuro a negro con la edad, de consistencia carbonácea quebradiza y hueca con la edad, con el margen lobulado. Peritecios de 1300-1500 × 500-900 $\mu \mathrm{m}$ de diámetro, ostíolos papilados. Ascas de $500 \times 10-14 \mu \mathrm{m}$, cilíndricas, con poro apical de 6,4-6,8 $\times$ 3,5-5,6 $\mu \mathrm{m}$, amiloide, octospóricas. Ascosporas de 40-48 × 9-14 $\mu \mathrm{m}$, fusoides, de color marrón oscuro a negras, con línea germinal longitudinal recta y lisa.

Material examinado. 29 octubre 2015, R. Soto-Agudelo 12 (HUQ); 29 diciembre 2015, R. Valenzuela 16406; $T$. Raymundo 5904 (ENCB).

Distribución ecológica y geográfica. Esta especie ha sido reportada sobre monocotiledóneas como bambú y palmas, y en este trabajo sobre raíz de Guadua angustifolia. Citada de Brasil, Ecuador, Estados Unidos, Guayana Francesa, Honduras, Malasia, México, Nueva Zelanda y Taiwán (Rogers y Ju, 1998; Medel y Chacón, 1997 como Ustulina pavimentosa (Ces.) Cooke) y Cuba (RecioHerrera y Maldonado-González, 2014). Se registra por primera vez para Colombia.

**Nummularia diatrypeoides Rehm, Annls Mycol. 5(6): 527, 1907. Figura $4 \mathbf{Y}$

Estroma de 1-10 mm de diámetro y hasta $1 \mathrm{~mm}$ de alto, circular a discoide, errumpente, plano, de color negro brillante, consistencia carbonosa. Peritecios de 700-800 $\times$ 300-450 $\mu \mathrm{m}$, ovoides. Ostíolos evidentes abriendo de manera puntuada. Ascas clavadas, cortamente estipitadas, con poro apical inamilode en forma de corona, octospóricas. Ascosporas de 42-56 × 24-32 $\mu \mathrm{m}$, elipsoidales, marrón oscuro, con poro germinativo recto, lisas.

Material examinado. 29 diciembre 2015, T. Raymundo 5933 (ENCB).

Distribución ecológica y geográfica. Esta especie crece en ramas de árboles en descomposición. Fue citada de Brasil por Rhem (1907) sobre ramas caídas. Ju, et al. (1998) al estudiar el género Biscogniauxia, encontraron que la mayoría de las especies de Nummularia pertenecían a este género; sin embargo, al estudiar el espécimen tipo de Nummularia diatrypeoides encontraron que no se trataba de una Biscogniauxia y coincidieron con lo señalado por Martin en 1961 (fide Ju, et al., 1998), que la señalaba como una verdadera Nummularia, pero con posición taxonómica incierta y se requiere de un análisis molecular para definir bien este taxón. La descripción del material estudiado de Colombia coincide con la dada por Ju, et al. (1998) y se considera como la primera cita para el país.

**Rosellinia corticium (Schwein.) Sacc. Syll. fung. (Abellini) 1: 253, 1882. Figura 4Z

Peritecios solitarios a gregarios, de 500-1500 $\mu \mathrm{m}$ de diámetro, globosos, negros, papilados, gregarios sobre un subiculum blanquecino, algodonoso. Ascas de 148-208 $\times$ 6-6,4 $\mu \mathrm{m}$, cilíndricas, uniseriadas, con poro apical amiloide, octospóricas. Ascosporas de 19-27× 7,5-10 $\mu$ m, elipsoidales, inequiláteras, con los extremos redondeados, marrón pálido a oliváceas, con poro germinal recto, lisas.

Material examinado. 29 diciembre 2015, R. SotoAgudelo 71 (HUQ).

Distribución ecológica y geográfica. Esta especie ha sido registrada sobre monocotiledóneas y en esta ocasión sobre Guadua angustifolia en descomposición. Citada de Europa y Asia (Petrini, 1992), México (San Martín y Rogers, 1995) y Taiwán (Ju y Rogers, 1999). Se registra por primera vez para Colombia.

**Xylaria bambusicola Y.M. Ju \& J.D. Rogers, Mycotaxon 73: 400, 1999. Figura 4ZA

Estromas densamente gregarios, de 20-60 × 1,0-2,5 $\mathrm{mm}$, cilíndricos a clavados, con el ápice agudo y estéril, de color marrón oscuro o negro con la edad, frágil, liso con algunas papilas ostiolares evidentes, de consistencia carbonosa. Peritecios de 300-500 $\mu \mathrm{m}$ de diámetro, globosos, con pared gruesa. Ascas de 120-150 × 5-7 $\mu \mathrm{m}$, cilíndricas, octospóricas, con poro apical de 2,4-3 × 2,5$2,8 \mu \mathrm{m}$, en forma de sombrero, amiloides. Ascosporas de 

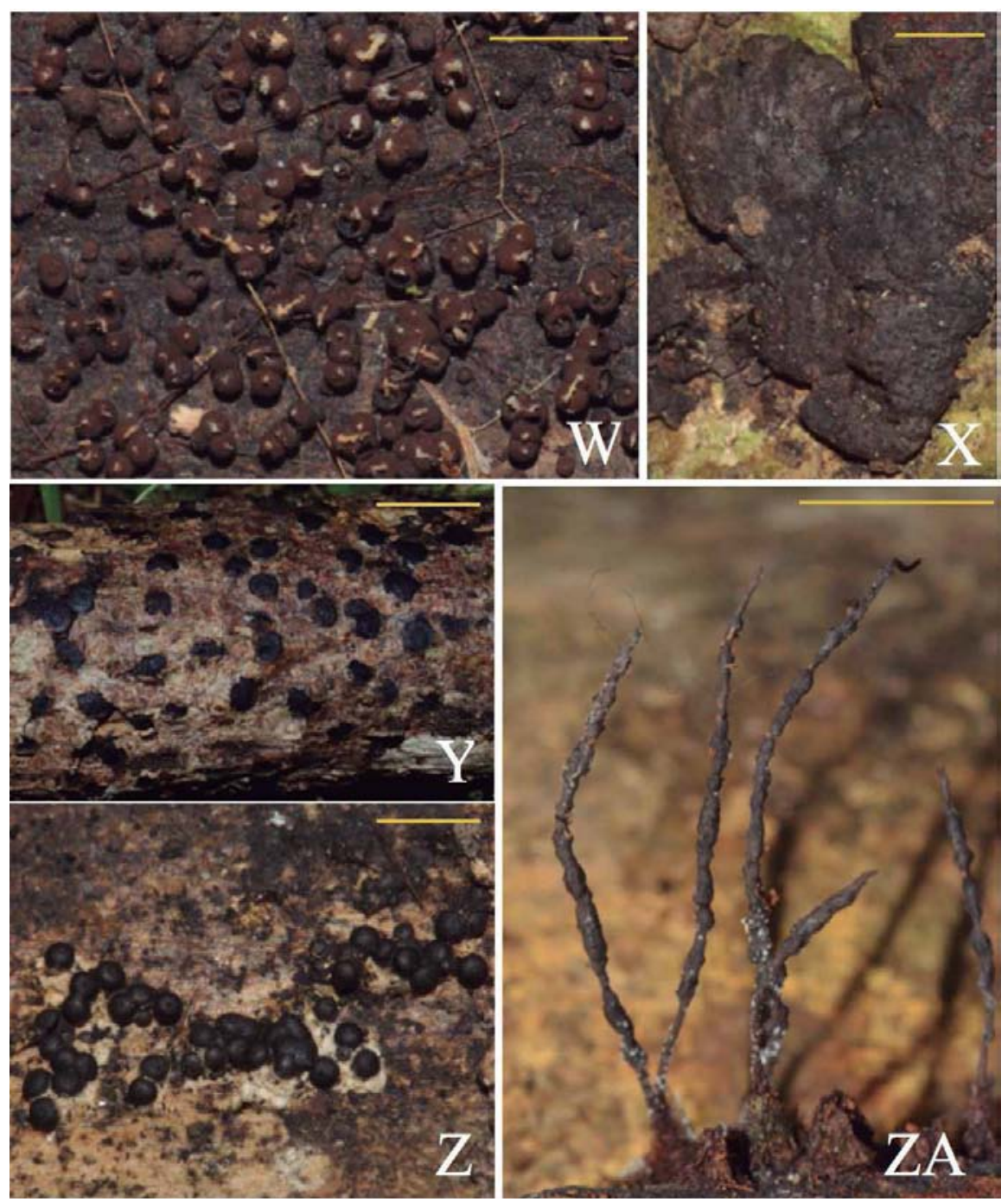

Figura 4. W-ZA. W. H. leneormandii, X. Kretzschmaria pavimentosa, Y. Nummularia diatrypeoides, Z. Rosellinia corticium, ZA. Xylaria bambusicola. (escala $=2 \mathrm{~cm})$.

9-12 × 4-5 $\mu \mathrm{m}$, elipsoidales, inequiláteras con los extremos redondeados, lisas y con poro germinal longitudinal recto a lo largo de la espora.

Material examinado. 29 diciembre 2015, R. SotoAgudelo 64, 68 (HUQ); R. Valenzuela 16411, 16418 (ENCB); T. Raymundo 5924, 5928 (ENCB).

Distribución ecológica y geográfica. Esta especie se había registrado sobre Bambusa oldhamii y Phyllostachys pubescens en Taiwán y en Colombia se observó abundante sobre raíz y tallos en descomposición de Guadua angustifolia. Citada de Taiwán por Ju y Rogers (1999). Se registra por primera vez para Colombia.

*Xylaria cubensis (Mont.) Fr., Nova Acta R. Soc. Scient., Ser. 3, 1: 126, 1851.

Material examinado. 29 diciembre 2015, T. Raymundo 5906 (HUQ, ENCB).

Distribución ecológica y geográfica. Esta especie crece sobre madera en descomposición de árboles en los guaduales. En Colombia fue citada de Caquetá por Chardon y Toro en 1930 (fide Vasco-Palacios y FrancoMolano, 2013) y del Parque Nacional Natural Los Nevados por Boekhout y Pulido (1989) y Pulido y Boekhout (1989). En el presente trabajo se cita por primera vez del Quindío.

**Xylaria grammica (Mont.) Mont., Nova Acta R. Soc. Scient., Ser. 3, 1: 128, 1851.

Estroma claviforme con el ápice redondeado a agudo y estéril, superficie gris-blanquecina, de color marrón obscuro cuando joven y con estrías o agrietado blanquecinas longitudinales con los ostiolos inmersos. Peritecios de 400$600 \mu \mathrm{m}$ de diámetro, globosos con pared gruesa. Ascas de 148-160 × 5-7 $\mu \mathrm{m}$, cilíndricas, con poro apical amiloide, octospóricas. Ascosporas de 10-12 × 4,5-5,5 $\mu \mathrm{m}$, elipsoidales, inequiláteras, lisas y con poro germinal longitudinal.

Material examinado. 29 diciembre 2015, T. Raymundo 5905 (HUQ, ENCB). 
Distribución ecológica y geográfica. Esta especie crece sobre madera en descomposición de árboles en los guaduales. Citada de Argentina, Cuba, Paraguay (Dennis, 1956) y México (San Martín y Rogers, 1989; Medel, Castillo y Guzmán, 2008). Se cita por primera vez para Colombia.

\section{Conclusiones}

De las 29 especies estudiadas 15 se encontraron creciendo sobre Guadua angustifolia, lo que equivale al 51,7\% por lo que el bambú colombiano representa un sustrato de gran importancia para el desarrollo de estos organismos. Los guaduales han sido inducidos en muchas localidades de Colombia para su explotación comercial y no son la excepción los estudiados en el presente trabajo, por lo que la vegetación original fue desplazada (bosque tropical seco y bosque de niebla), y los hongos que crecían sobre ésta, encontraron en Guadua angustifolia un excelente sustrato para ser colonizado por varias especies de ascomicetos y basidiomicetos que crecían en la vegetación original. No obstante, los estudios de la micobiota en los guaduales son escasos y hasta ahora no se habían mencionado registros de ascomicetos sobre esta planta, por lo que el presente trabajo contribuye al conocimineto sobre la dinámica de las poblaciones fúngicas de los guaduales. La familia Xylariaceae fue la mejor representada con 12 especies, y los géneros con mayor número de especies fueron Hypoxylon y Xylaria con tres especies cada una. Es importante mencionar que el alto número de registros nuevos para el país son un buen indicador para continuar con el estudio de los ascomicetos asociados a los guaduales del Departamento del Quindío y de Colombia.

\section{Agradecimientos}

Los autores agradecen a las autoridades de la Reserva Natural Montaña del Ocaso y de la Universidad del Quindío por el apoyo y las facilidades otorgadas para la elaboración del presente trabajo. Valenzuela reconoce el apoyo de la COFAA e IPN en sus investigaciones, además a los proyectos SIP-20150540, SIP-20161164. Raymundo agradece a los proyecto SIP-20151530 y 20161166 del Instituto Politécnico Nacional.

\section{Conflicto de intereses}

Los autores declaran no tener conflicto de intereses.

\section{Referencias}

Agudelo, C.A. \& Gómez, G.D. 2001. Reserva Natural La Montaña del Ocaso: un nuevo modelo de conservación. (pp.26-41) En: Monografias de la flora andina. Importancia de la microcuenca del río Roble. Armenia: Conceptos gráficos Ltda. Universidad del Quindío.

Barr, M.E. 1984. Herpotrichia and its segregates. Mycotaxon, 20: $1-38$.

Betancour, M.A. Calderon, M.H., Betancourt, O.G. \& Sucerquia, A. G. 2007. Hongos macromycetes en dos relictos de bosque húmedo tropical montano bajo de la vereda la cuchilla, Marmato, Caldas. Boletín Cientifico del Museo de Historia Natural, Universidad de Caldas 11: 19-31.
Boekhout, T. \& Pulido, M. 1989. The occurrence of macrofungi and their habitats in vegetations along the Parque Los Nevados transect, in Van der Hammen T, Díaz-P. S, Alvarez VJ (eds.) La cordillera central colombiana transecto Parque Los Nevados (Segunda. Parte). Berlin: J. Cramer; Estudios de Ecosistemas Tropandinos. 3.

Breitenbach, J. \& Kränzlin, F. 1984. Champignons de Suisse I. Les Ascomycetes. Ed. Breitenbach y Kränzlin. Lucern.

Carmona, A., Fournier, J., Williams, C. \& Piepenbring, M. (2009). New records of Xylariaceous from Panama. North American Fungi. 4 (3): 1-11.

Chacón-Zapata, S. \& Tapia-Padilla, F. 2013. Algunas especies del género Byssosphaeria (Melanommataceae, Pleosporales) de Veracruz, México. Revista Mexicana de Biodiversida. 84: 739-745.

Chacón, S., Dörge, D., Weisenborn, J. \& Piepenbring, M. 2013. A new species and a new record of Diatrypaceae from Panama. Mycologia. 105: 681-688.

Chacón, S., Tapia, F., Esqueda, M. 2014. New records of Dothideomycetes from Mexico. Mycotaxon. 128: 145-157.

Chaverri, P., Huhndorf, S., Rogers, J.D. \& Samuels, G.J. 2011. Microhongos comunes de Costa Rica y otras regiones tropicales. $1^{\text {a }}$ ed. INBIO. Costa Rica.

Chen, C.Y. \& Hsieh, W.H. 2004a. Astrosphaeriella from Taiwan, including two new species. Bot. Bull. Acad. Sin. 45: 171-178.

Chen, C.Y. \& Hsieh, W.H. 2004b. Byssosphaeria and Herpotrichia from Taiwan, with notes on the taxonomic relationship between these two genera. Sydowia, 56: 24-38.

Dennis, R.W.G. 1956. Some Xylarias of tropical America. Kew Bulletin. 11: 401-444.

Fernández, F.A. \& Huhndorf, S.M. 2005. New species of Chaetosphaeria, Melanopsammella and Tainosphaeria gen. nov. from the Americas. Fungal Diversity. 18: 15-57.

Franco-Molano, A.E., Vasco-Palacios, A.M., López-Quintero, C. \& Boekout, T. 2005. Macrohongos de la región medio Caquetá. Guía de Campo. Multimpresos. Medellín.

Gallo, M:C., 1,2, Robledo, G., Romero, A.I. \& Catania, M.V. 2014. New records of Ascomycota in the northwestern Argentinean Yungas. Check List. 10 (3): 621-631.

Guu, J.R., Ju, Y.M. \& Hsieh, H.H. 2007. Nectriaceous fungi collected from forests in Taiwan. Botanical Studies. 48: 187-203.

Guu, J.R., Ju, Y.M. \& Hsieh, H.H. 2010. Bionectriaceous fungi collected from forests in Taiwan. Botanical Studies. 51: 61-74.

Guzmán, G., Torres, M., Ramírez-Guillen, F. \& Ríos-Hurtado, A. 2004. Introducción a los macromicetos del Chocó, Colombia. Revista Mexicana de Micología. 19: 33-43.

Herrera, S.C., Rossman, A.Y., Samuels, G.J., Liparini P.O. \& Chaverri, P. 2015. Systematics of the Cosmospora viliuscula species complex. Mycologia. 107 (3): 532-557.

Hirooka, T. \& T. Kobayashi. 2007. Taxonomic studies of nectrioid fungi in Japan. I: The genus Neonectria. Mycoscience. 48: 53-62.

Iturriaga, T. \& Pfister, D.H. 2006. A monograph of the genus Cookeina (Ascomycota, Pezizales, Sarcoscyphaceae). Mycotaxon: 95: 137-180.

Ju, Y.M. \& Rogers, J.D. 1996. A revision of the genus Hypoxylon. Mycologia Memoir no. 20. APS Press, St. Paul, MN. 365 pp.

Ju, Y.M. \& Rogers, J.D. 1999. The Xylariaceae of Taiwan (excluding Anthostomella). Mycotaxon. 73: 343-440. 
Ju, Y.M., Rogers, J.D., San Martín, F. \& Granmo, F. 1998. The genus Biscogniauxia. Mycotaxon. 66: 1-98.

Kirk, P.M., Canon, P.F., Minter, D.W., \& Stalpers, J.A. 2008. Ainsworth \& Bisby's Dictionary of the Fungi. 10 ed. International Mycological Institute, CAB International, Wallingford. $784 \mathrm{p}$.

Li, W.Y. \& Zhuang, W.Y. 2008. Notes on the genus Byssosphaeria (Melanommataceae) from China. Mycosystema. 27: 48-53.

Liu, J.L., Phookamsak, R., Jones, E.B.G., Zhang, Y., Ko-Ko, T.W., Hu, H.L., Boonmee, S., Doilom, M., Chukeatirote, E., Bahkali, A.H., Wang, Y. \& Hyde, K.D. 2011. Astrosphaeriella is polyphyletic, with species in Fissuroma gen. nov., and Neoastrosphaeriella gen. nov. Fungal Diversity. 51: 135-154.

Medel, R. y S. Chacón. 1997. Ascomycetes poco conocidos de México VIII. Algunas especies del bosque mesófilo de Veracruz. Acta Botánica Mexicana. 39: 43-52.

Medel, R., Castillo, R. \& Guzmán, G. 2008. Las especies de Xylaria (Ascomycota, Xylariaceae) conocidas de Veracruz, México y discusión de nuevos registros. Revista Mexicana de Micología. 28: 101-118.

Méndez-Mayboca, F., Checa, J., Esqueda, M. \& Chacón, S. 2010. New records of Loculoascomycetes from natural protected areas in Sonora, Mexico. Mycotaxon. 111: 19-30.

Moreno-M, L.E., Trujillo, E.E., \& Osorio, L.R. 2007. Estudio de las características físicas de haces de fibra de Guadua angustifolia. Scientia et Technica Año XIII. 34: 613-617.

Murillo, C., Albertazzi, F.J., Carranza, J., Lumbsch, H.T. \& Tamayo G. 2009. Molecular data indicate that Rhytidhysteron rufulum (Ascomycetes, Patellariales) in Costa Rica consists of four distinct lineages corroborated by morphological and chemical characters. Mycological Research. 113: 405-416.

Petrini, L. E. 1992. Rosellinia species of the temperate zones. Sydowia. 44: 169-281.

Pulido, M. \& Boekhout, T. 1989. Distribution of macrofungi along The Parque Los Necados transect. 485-505, inVan der Hammen, T. S. Díaz y V. Alvarez (eds.). La cordillera Central Colombiana transecto Parque Los Nevados (segunda parte). Studies on Tropical Andean Ecosystems, Volume 3.

Ramírez-Díaz, F. \& Camargo, J.C. 2015. Diversidad vegetal de los bosques de guadua en el Eje Cafetero de Colombia. Recursos Naturales y Ambiente, 65-66: 21-25.
Rappaz, F. 1987. Taxonomie et nomenclature des diatrypacées à ascas octosporés I. Mycologia Helvetica. 2: 285-648.

Raymundo, T., Escudero-Leyva, E., Ortega-López, I., CastroBustos, D., León-Avendaño, H. \& Valenzuela, R. 2014. Ascomicetos del Bosque tropical caducifolio en el Parque Nacional Lagunas de Chacahua, Oaxaca, México. Boletín de la Sociedad Micológica de Madrid. 34: 9-21.

Recio-Herrera G.M. \& Maldonado-González, S.G. 2014. A revision of the genus Kretzschmaria (Ascomycota, Xylariaceae) in Cuba. Willdenowia. 44: 57-64.

Restrepo-F., G.M., Vélez-A., P.E., Botero-A., P.A., \& Pulido-V., C. 2005. Reconocimiento de macromicetos asociados al cultivo de Guadua angustifolia en Caldas, Colombia. Manejo Integrado de Plagas y Agroecología (Costa Rica). 76: 25-31.

Réblová, M. 1997. Revision and reclassification of some Chaetosphaeria species. Czech Mycology. 50: 73-83.

Rehm, H. 1907. Ascomycetes novi. Annales Mycologici. 5: 516-546.

Rogers, J.D. \& Ju, Y.M. 1998. The genus Kretzschmaria. Mycotaxon. 68: 345-393.

Rogers, J.D., San Martín, F. \& Ju, Y.M. 2002. Three new taxa of Camillea from Costa Rica. Sydowia. 54 (1): 84-90.

Samuels, G.J. \& Müller, E. 1979. Life-history studies of Brazilian Ascomycetes. 7. Rhytidhysteron rufulum and the genus Eutryblidiella. Sydowia. 32: 277-292.

Samuels, G.J. \& Dumont, K.P. 1982. The genus Nectria (Hypocreaceae) in Panama. Caldasia. 13: 379-423.

San Martín, F. \& Rogers, J.D. 1989. A preliminary of account of Xylaria of Mexico. Mycotaxon. 34 (2): 283-374.

San Martín, F. \& Rogers, J.D. 1995. Rosellinia y Thamnomyces in Mexico. Mycotaxon. 53: 115-127.

San Martín, F., Ju, Y.M. \& Rogers, J.D. 1999. Algunas especies de Hypoxylon (Pyrenomycetes, Xylariaceae) de México. Acta Botanica Mexicana. 47: 31-53.

Stadler, M., Laessoe, T., Decock, C., Schimieschek, B., Tichy, H.V. \& Persoh, D. 2014. A polyphasic taxonomy of Daldinia (Xylariaceae). Studies in Mycology. 77: 1-143.

Vasco-Palacios, A.M., \& Franco-Molano, A.E., López-Quintero, C. \& T. Boekhout, T. 2005. Macromicetes (Ascomycota, Basidiomycota) de la región del medio Caquetá, departamento de Caquetá y Amazonas (Colombia). Biota Colombiana. 6: 27-159.

Vasco-Palacios, A.M., \& Franco-Molano, A.E. 2013. Diversity of Colombian macrofungi (Ascomycota-Basidiomycota). Mycotaxon 121: 99-504. 